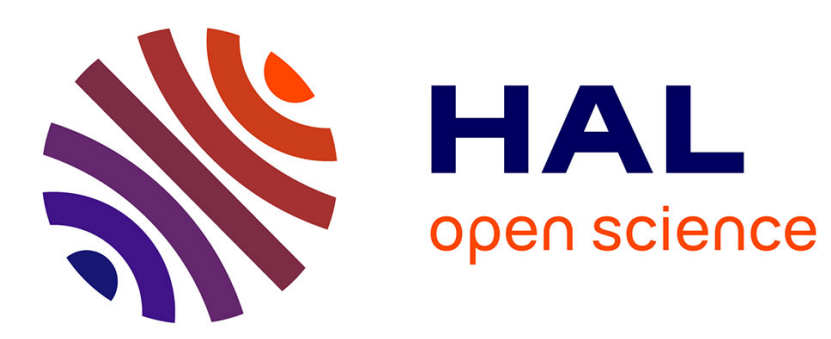

\title{
An exact spectral representation of the wave equation for propagation over a terrain
}

Alexandre Chabory, Christophe Morlaas, Rémi Douvenot, Bernard Souny

\section{To cite this version:}

Alexandre Chabory, Christophe Morlaas, Rémi Douvenot, Bernard Souny. An exact spectral representation of the wave equation for propagation over a terrain. ICEAA 2012, International Conference on Electromagnetics in Advanced Applications, Sep 2012, Cape Town, South Africa. pp 717-720, 10.1109/ICEAA.2012.6328722 . hal-01022309

\section{HAL Id: hal-01022309 \\ https://hal-enac.archives-ouvertes.fr/hal-01022309}

Submitted on 22 Sep 2014

HAL is a multi-disciplinary open access archive for the deposit and dissemination of scientific research documents, whether they are published or not. The documents may come from teaching and research institutions in France or abroad, or from public or private research centers.
L'archive ouverte pluridisciplinaire HAL, est destinée au dépôt et à la diffusion de documents scientifiques de niveau recherche, publiés ou non, émanant des établissements d'enseignement et de recherche français ou étrangers, des laboratoires publics ou privés. 


\title{
An Exact Spectral Representation of the Wave Equation for Propagation Over a Terrain
}

\author{
A. Chabory, C. Morlaas, R. Douvenot, and B. Souny *
}

\begin{abstract}
An exact spectral representation of the wave equation above a dielectric ground is proposed. The formulation is based on the diagonalisation of the vertical operator, takes into account the angle-dependance of the reflexion coefficient, and does not include any paraxial approximation. The expressions of the spectrum comprise two parts: a continuous part and a discrete part. The latter corresponds to a possible surface wave. The use of this result in split-step algorithms to simulate wave propagation requires a discretization of the spectrum. To render the discretization consistent, an alternative discrete spectral representation is proposed that intrinsicaly includes the truncation of the computation domain at a finite high.
\end{abstract}

\section{INTRODUCTION}

To model the propagation of electromagnetic waves over the ground at large distances, one can rely on split-step methods based on the parabolic approximation of the wave equation [1]. The computation is performed step by step at increasing distances, going back and forth from a spatial to a spectral representation of the wave. Such methods can generally take into account the terrain profile, a possible ground wave, and the electrical characteristics of the atmosphere.

To model a ground characterized by a constant surface impedance, the continuous mixed Fourier transform has been proposed [2]. This transform matches the spectral representation to an impedance boundary condition via a change of variable. A discretized counterpart of this transform, the discrete mixed Fourier transform (DMFT), has been developed to render the scheme self consistent and avoid numerical instabilities.

In many applications, e.g. for rough surfaces, a constant impedance may not be sufficient to model the boundary condition at the ground level because the reflexion coefficient depends on the angle of incidence. To deal with this difficulty, Kuttler and Dockery have proposed to keep the impedance constant at a given range, but to extract the impedance value from the dominant propagation direction [2]. Janaswamy has proposed a more rigourous solution to model the propagation over

*ENAC/Telecom Lab/EMA, 7 av E. Belin, 31055 Toulouse, France, e-mail: chabory@recherche.enac.fr, tel.: +33 562174325, fax: +33562174270 . a nonconstant immittance plane [3], i.e. with an angle-dependant reflexion coefficient.

In this article, we theoretically develop an exact spectral representation of the vertical operator taking into account the angle dependance of the reflexion coefficient. We directly start from Maxwell equations, i.e. without the parabolic approximation. The demonstration is directly founded on the diagonalisation of the vertical operator [4]. Besides, in order to avoid the numerical difficulties that may arise when discretizing a continuous spectral representation, we introduce an alternative formulation for a domain of finite high. Thus, this formulation includes the necessary truncation of the computation domain at a finite altitude. We show that this leads to a discrete spectrum.

This article is organized as follows. In Section 2 we present the configuration. The spectral representation is demonstrated in Section 3. Next, in Section 4 we modify this representation to account for a domain of finite high before presenting the application of the method in Section 5.

\section{CONFIGURATION}

We consider a time-harmonic configuration where the fields are transverse magnetic (TM) with respect to the vertical axis $z$. Note that the transverse electric case can be analyzed in a similar way. Let $(r, \phi, z)$ be the local cylindrical coordinate system with unit vectors $(\hat{\mathbf{r}}, \hat{\phi}, \hat{\mathbf{z}})$. We assume a rotationnal symmetry about the vertical axis. The ground/atmosphere are characterized by a constant permeability $\mu_{0}$ and by space-varying permittivity $\varepsilon_{r}(r, z)$ and conductivity $\sigma(r, z)$ The problem can be formulated from a vector potential $\boldsymbol{\Pi}_{\mathrm{e}}$ such that

$$
\begin{aligned}
& \mathbf{E}=\boldsymbol{\Pi}_{\mathrm{e}}-\nabla \frac{1}{k^{2}} \nabla \cdot \boldsymbol{\Pi}_{\mathrm{e}}, \\
& \mathbf{H}=\frac{1}{-j \omega \mu_{0}} \nabla \times \boldsymbol{\Pi}_{e} .
\end{aligned}
$$

where $k=-j \omega \mu_{0}(\sigma+j \omega \varepsilon)$ is the wavenumber. 


\section{HOMOGENEOUS ATMOSPHERE ABOVE A DIELECTRIC GROUND}

\subsection{Formulation}

For an homogeneous atmosphere and ground, upon replacing $\Pi_{\mathrm{e}}$ by $\psi \hat{\mathbf{z}}$, the wave equation can be cast in cylindrical coordinates as

$$
-\frac{1}{r} \frac{\partial}{\partial r}\left(r \frac{\partial}{\partial r} \Psi\right)-\frac{\partial^{2}}{\partial z^{2}} \Psi-k^{2}(z) \Psi=0,
$$

with $r \in] 0, \infty[, z \in \mathbb{R}$. Furthermore

$$
k(z)= \begin{cases}k_{a} & z>0, \\ k_{g} & z<0,\end{cases}
$$

with $k_{a}$ and $k_{g}$ the wavenumbers in the atmosphere and ground, respectively. At infinity, $\Psi$ is subjected to radiating boundary conditions. At the ground level $z=0$, the boundary conditions is imposed by the ground/atmosphere interface.

\subsection{Principle of the determination of the vertical spectral representation}

The variables $r$ and $z$ are separate in (2). Consequently, we introduce an operator $L_{z}$ acting on the vertical coordinate $z$ such that

$$
L_{z} \Psi=-\left(\frac{\partial^{2}}{\partial z^{2}}+k^{2}(z)\right) \Psi .
$$

This operator can be associated with a SturmLiouville problem of the third kind. The spectral representation is introduced via the diagonalisation of this operator.

To do so, we employ the method developed in [4], that can be divided in 3 steps:

- We determine the Green's function $G\left(z, z^{\prime}, \lambda\right)$ of the operator $L_{z}-\lambda I$, with $\lambda \in \mathbb{C}, I$ the identity operator, and $\left(z^{\prime}, z\right)$ the position of the source and observation, respectively.

- We write the following identity, demonstrated in [4],

$$
\frac{1}{2 j \pi} \lim _{R \rightarrow \infty} \oint_{\mathcal{C}_{R}} G\left(z, z^{\prime}, \lambda\right) d \lambda=-\delta\left(z-z^{\prime}\right),
$$

where $\mathcal{C}_{R}$ is the circle centered at 0 of radii $R$ in the complex $\lambda$-plane. Then, we evaluate explicitly the integral in (5) taking care of the contributions of branch cuts and poles.

- Finally, we write

$$
\Psi(r, z)=\int_{0}^{\infty} \delta\left(z-z^{\prime}\right) \Psi\left(r, z^{\prime}\right) d z^{\prime} .
$$

Using (5), we can substitute $\delta\left(z-z^{\prime}\right)$ by the explicit contributions of the branch cuts and poles. The expression that we obtain is the sprectral representation of the operator.

\subsection{Green's function}

The Green's function of $L_{z}-\lambda I$ is the solution of

$$
-\frac{\partial}{\partial z^{2}} G\left(z, z^{\prime}, \lambda\right)-\left(k^{2}+\lambda\right) G\left(z, z^{\prime}, \lambda\right)=\delta\left(z-z^{\prime}\right),
$$

for $\left(z, z^{\prime}\right) \in \mathbb{R}^{2}$. Besides, at $z \rightarrow+\infty, G$ is subjected to radiation boundary conditions. At $z=0$, a reflexion coefficient is introduced to restrict the computation to the domain $z \geq 0$ and $z^{\prime} \geq 0$, because in this article we are only interested by the field in the atmosphere. Using a classical method [4] for the determination of Green's functions, we obtain

$$
G\left(z, z^{\prime}, \lambda\right)=\frac{e^{-j k_{z a}\left|z-z^{\prime}\right|}+\Gamma e^{-j k_{z a}\left(z+z^{\prime}\right)}}{2 j k_{z a}},
$$

for $z \geq 0$ and $z^{\prime} \geq 0$, with $k_{z a}=\sqrt{k_{a}^{2}+\lambda}$. The suitable determination of the square root is the one that respects the radiation condition, i.e. $\operatorname{Im}\left(k_{z a}\right) \leq 0$. The reflexion coefficient is given by

$$
\Gamma=\frac{Z_{a}-Z_{g}}{Z_{a}+Z_{g}},
$$

with

$$
Z_{a}=\frac{j k_{z a}}{\sigma_{a}+j \omega \varepsilon_{a}}, \quad Z_{g}=\frac{j k_{z g}}{\sigma_{g}+j \omega \varepsilon_{g}} .
$$

Note that the condition at $z=0$ corresponds in the spectral domain to the following impedance boundary condition

$$
\frac{\partial}{\partial z} G\left(z, z^{\prime}, \lambda\right)-j k_{z a} \frac{1-\Gamma}{1+\Gamma} G\left(z, z^{\prime}, \lambda\right)=0,
$$

inside which the impedance depends on the spectral variable $k_{z a}$. This condition can be obtained by solving the equation in the complete domain $z \in$ $\mathbb{R}$. Thus, it is an exact formulation for the ground interface.

\subsection{Integration in the complex $\lambda$-plane}

We now have to evaluate the integral in (5). In the complex $\lambda$-plane, the square root in $k_{z a}$ introduces a branch cut. As indicated in Fig. 1, we define a new contour $\mathcal{C}_{a}$ to add the contribution of the branch cut to the integral.

We note that on the lower part of $\mathcal{C}_{a}, k_{z a}$ is real and positive, while on the upper part $k_{z a}$ is real 


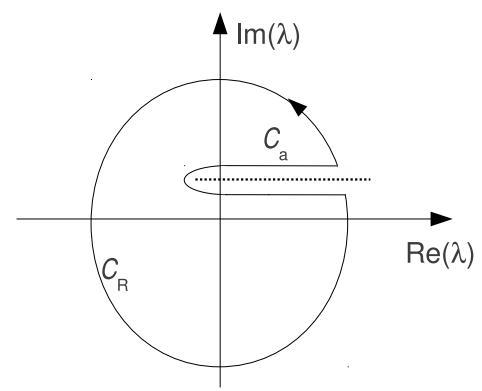

Figure 1: Contours of integration in the complex $\lambda$-plane.

and negative. A change of variable $\lambda \leftrightarrow k_{z a}$ in the integral of $G\left(z, z^{\prime}, \lambda\right)$ over both parts of $\mathcal{C}_{a}$ yields

$$
\begin{array}{r}
\int_{\mathcal{C}_{a}} G\left(z, z^{\prime}, \lambda\right) d \lambda=\int_{0}^{+\infty}\left(e^{j k_{z a} z}+\Gamma e^{-j k_{z a} z}\right) \\
\cdot\left(e^{j k_{z a} z^{\prime}}+\Gamma e^{-j k_{z a} z^{\prime}}\right) \frac{j}{\Gamma} d k_{z a}
\end{array}
$$

To evaluate the integral in (5), we also have to consider the possible presence of poles inside the contour $\mathcal{C}_{R}$. Note that $G$ is regular when $\lambda \rightarrow k_{a}^{2}$ despite the factor $1 / k_{z a}$ in (8). Thus, the only poles we have to consider in $G$ are the poles of $\Gamma$. From (9), if $\Gamma$ has a pole, its expression is given by

$$
\lambda^{p}=\frac{k_{a}^{2}}{1+\epsilon},
$$

where

$$
\epsilon=\frac{\sigma_{a}+j \omega \epsilon_{a}}{\sigma_{b}+j \omega \epsilon_{b}}
$$

The residue of this pole corresponds to

$$
\operatorname{Res}\left(G, \lambda^{p}\right)=\frac{-2 j k_{z a}^{p}}{1-\epsilon^{2}} e^{-j k_{z a}^{p}\left(z+z^{\prime}\right)}
$$

with

$$
k_{z a}^{p}=\sqrt{k_{a}^{2} \frac{\epsilon}{1+\epsilon}} .
$$

Finally, if the contribution of the branch cut (12) and the pole (15) are taken into account, the expression (5) explicitly results in

$$
\begin{array}{r}
\delta\left(z-z^{\prime}\right)=\frac{2 j k_{z a}^{p}}{1-\epsilon^{2}} e^{-j k_{z a}^{p}\left(z+z^{\prime}\right)} \\
+\frac{1}{2 \pi} \int_{0}^{+\infty} \frac{1}{\Gamma}\left(e^{j k_{z a} z^{\prime}}+\Gamma e^{-j k_{z a} z^{\prime}}\right) \\
\cdot\left(e^{j k_{z a} z}+\Gamma e^{-j k_{z a} z}\right) d k_{z a} .
\end{array}
$$

\subsection{Spectral representation}

Substituting (17) in (6), and interchanging the order of integration, we end up with the spectral representation

$$
\begin{aligned}
& \Psi(r, z)=\tilde{\Psi}^{p}(r) e^{-j k_{z a}^{p} z}+ \\
& \quad \int_{0}^{+\infty} \tilde{\Psi}\left(r, k_{z}\right)\left(e^{j k_{z} z}+\Gamma\left(k_{z}\right) e^{-j k_{z} z}\right) d k_{z}
\end{aligned}
$$

with

$$
\begin{aligned}
\tilde{\Psi}^{p}(r)= & \frac{2 j k_{z a}^{p}}{1-\epsilon^{2}} \int_{0}^{+\infty} \Psi(r, z) e^{-j k_{z a}^{p} z} d z \\
\tilde{\Psi}\left(r, k_{z a}\right)= & \frac{1}{2 \pi} \int_{0}^{+\infty} \frac{\Psi(r, z)}{\Gamma\left(k_{z a}\right)} \\
& \cdot\left(e^{j k_{z a} z}+\Gamma\left(k_{z a}\right) e^{-j k_{z a} z}\right) d z .
\end{aligned}
$$

The first term of (18) correponds to the contribution of the pole, and can be associated with a possible ground/surface wave. The second term is the continuous spectrum that represents plane waves and their reflexion over the ground.

The spectral representation has been obtained here for TM fields and for a ground modeled by a dielectric interface. Note that the formulation can be extended to other cases where the reflexion coefficient $\Gamma\left(k_{z a}\right)$ and its associated poles are known. This may include, for example, grounds characterized by a rough surface or by a multilayer dielectric slab.

\section{HOMOGENEOUS ATMOSPHERE OF FINITE HIGH}

In the previous spectral representation, the continuous spectrum comes from the fact that the domain is not bounded, i.e. $z \in[0,+\infty[$. However, for obvious numerical reasons, the computation domain must be bounded. We introduce in this Section a spectral representation for a domain of finite high. We show that this leads to a discrete spectrum.

The method is similar to the previous one except that there now exists a boundary condition at a finite high $z=h$ given by

$$
\frac{\partial}{\partial z} G\left(z, z^{\prime}, \lambda\right)-j k_{z a} \frac{1-\Gamma}{1+\Gamma} G\left(z, z^{\prime}, \lambda\right)=0 .
$$

The evaluation of the Green's function yields

$$
\begin{aligned}
G\left(z, z^{\prime}, \lambda\right) & =\frac{-1}{4 k_{z a} \Gamma\left(k_{z a}\right) \sin \left(k_{z a} h\right)} \\
& \left(e^{j k_{z a}\left(z^{\prime}-h\right)}+\Gamma\left(k_{z a}\right) e^{-j k_{z a}\left(z^{\prime}-h\right)}\right) \\
& \cdot\left(e^{j k_{z a} z}+\Gamma\left(k_{z a}\right) e^{-j k_{z a} z}\right)
\end{aligned}
$$


for $0<z<z^{\prime}$, and

$$
\begin{aligned}
& G\left(z, z^{\prime}, \lambda\right)=\frac{-1}{4 k_{z a} \Gamma\left(k_{z a}\right) \sin \left(k_{z a} h\right)} \\
& \cdot\left(e^{j k_{z a} z^{\prime}}+\Gamma\left(k_{z a}\right) e^{-j k_{z a} z^{\prime}}\right) \\
& \cdot\left(e^{j k_{z a}(z-h)}+\Gamma\left(k_{z a}\right) e^{-j k_{z a}(z-h)}\right)
\end{aligned}
$$

for $z^{\prime}<z<h$. Taking care of the contributions of the poles and branch cuts, the integration of $G$ over $\mathcal{C}_{R}$ in (5) leads to a discrete spectrum. Its expression is given by

$$
\begin{aligned}
\Psi(r, z) & =\tilde{\Psi}^{p}(r) e^{-j k_{z a}^{p} z} \\
& +\sum_{n=1}^{+\infty} \tilde{\Psi}_{n}(r)\left(e^{j \frac{n \pi}{h} z}+\Gamma_{n} e^{-j \frac{n \pi}{h} z}\right)
\end{aligned}
$$

with $\Gamma_{n}=\Gamma(n \pi / h)$. The spectral components can be expressed as

$$
\begin{aligned}
& \tilde{\Psi}^{p}(r)=\frac{2 j k_{z a}^{p}}{1-e^{-2 j k_{z a}^{p} h}} \frac{1}{1-\epsilon^{2}} \int_{0}^{h} \Psi(r, z) e^{-j k_{z a}^{p} z} d z \\
& \tilde{\Psi}_{n}(r)=\int_{0}^{h} \frac{\Psi(r, z)}{2 h \Gamma_{n}}\left(e^{j \frac{n \pi z}{h}}+\Gamma_{n} e^{-j \frac{n \pi z}{h}}\right) d z .
\end{aligned}
$$

\section{APPLICATION TO PROPAGATION SIMULATIONS}

\subsection{Homogeneous ground/atmosphere}

The previous spectral representation can be used to model the propagation in an homogeneous atmosphere above an homogeneous ground. We assume that $\Psi\left(r_{0}, z\right)$ is known. To obtain $\Psi$ at $r>r_{0}$, we determine both components of the spectral representation $\tilde{\Psi}^{p}\left(r_{0}\right)$ and $\tilde{\Psi}\left(r_{0}, k_{z a}\right)$ using the expressions in (24). Then, for $r>r_{0}, \tilde{\Psi}^{p}(r)$ and $\tilde{\Psi}\left(r, k_{z a}\right)$ are solutions of

$$
\frac{1}{r} \frac{\partial}{\partial r}\left(r \frac{\partial}{\partial r} \tilde{\Psi}\right)+\left(k_{a}^{2}-k_{z a}^{2}\right) \tilde{\Psi}=0
$$

If the field is propagating towards $r>r_{0}$, we can solve this equation. We end up with

$$
\tilde{\Psi}\left(r, k_{z}\right)=\tilde{\Psi}\left(r_{0}, z\right) \frac{H_{0}^{(2)}\left(k_{r} r\right)}{H_{0}^{(2)}\left(k_{r} r_{0}\right)},
$$

with $H_{0}^{(2)}$ the Hankel function of the second kind and of order 0 , and $k_{r}=\sqrt{k_{a}^{2}-k_{z a}^{2}}$ where $\operatorname{Im}\left(k_{r}\right) \leq 0$. Finally, using (18), we can obtain $\Psi(r, z)$ from $\tilde{\Psi}\left(r, k_{z}\right)$. Note that for $r-r_{0}$ greater than few wavelength, the Hankel functions can be simplified, and the expression is reduced to

$$
\tilde{\Psi}\left(r, k_{z}\right)=\tilde{\Psi}\left(r_{0}, z\right) e^{-j k_{r}\left(r-r_{0}\right)} .
$$

\subsection{General case}

To model the propagation, we have gone back and forth from a spatial to a spectral representation. Thus, there exists an analogy with classical methods based on the parabolic equation (PWE). However here no hypothesis on the paraxiality is made. Besides the variation of the reflexion coefficient with $k_{z a}$ (and thus with incidence) is intrinsicaly modeled. As with PWE, it is be possible to derive a more-general split-step algorithm that takes into account the terrain profile, and variations in the electrical characteristics. Details of such an algorithm are presented in [5].

\section{CONCLUSION}

We have proposed a spectral representation of TM fields in an homogeneous atmosphere above a dielectric ground that takes into account the angledependance of the reflexion coefficient. The spectral representation includes a continuous and a discrete part. The former corresponds to plane waves and their reflexion over the ground. The latter corresponds to a possible ground wave. This method can be extended to TE configurations and to any ground for which the reflexion coefficient and its poles are known.

An alternative discrete formulation has been proposed that includes a truncation of the domain at finite high. This formulation is suitable for being employed in split-step algorithms, for which the problem must be bounded in space and discretized.

\section{References}

[1] M. Levy, Parabolic Equation Methods for Electromagnetic Wave Propagation, ser. IEE electromagnetic waves series 45, IET, Ed., 2000.

[2] D. G. Dockery and J. R. Kuttler, "An improved impedance-boundary algorithm for Fourier split-step solutions of the parabolic wave equation," IEEE trans. Antennas Propag., vol. 44, no. 12, pp. 1592-1599, 1996.

[3] R. Janaswamy, "Radio wave propagation over a nonconstant immitttance plane", Radio Sci., vol. 36 , no. 3, pp.387-405, 2001.

[4] D. G. Dudley, Mathematical Foundations for Electromagnetic Theory, IEEE press, NewYork, 1994.

[5] R. Douvenot, C. Morlaas, A. Chabory, B. Souny, "Matrix split-step resolution for propagation based on an exact spectral formulation", in ICEAA, 2012. 